\title{
Engineering Escherichia coli FAB system using synthetic plant genes for the production of long chain fatty acids
}

\author{
Elias Kassab, Monika Fuchs, Martina Haack, Norbert Mehlmer and Thomas B. Brueck ${ }^{*}$ (D)
}

\begin{abstract}
Background: Sustainable production of microbial fatty acids derivatives has the potential to replace petroleum based equivalents in the chemical, cosmetic and pharmaceutical industry. Most fatty acid sources for production oleochemicals are currently plant derived. However, utilization of these crops are associated with land use change and food competition. Microbial oils could be an alternative source of fatty acids, which circumvents the issue with agricultural competition.

Results: In this study, we generated a chimeric microbial production system that features aspects of both prokaryotic and eukaryotic fatty acid biosynthetic pathways targeted towards the generation of long chain fatty acids. We redirected the type-II fatty acid biosynthetic pathway of Escherichia coli BL21 (DE3) strain by incorporating two homologues of the beta-ketoacyl-[acyl carrier protein] synthase I and II from the chloroplastic fatty acid biosynthetic pathway of Arabidopsis thaliana. The microbial clones harboring the heterologous pathway yielded $292 \mathrm{mg} / \mathrm{g}$ and 220 mg/g DCW for KAS I and KAS II harboring plasmids respectively. Surprisingly, beta-ketoacyl synthases KASI/II isolated from A. thaliana showed compatibility with the FAB pathway in E. coli.
\end{abstract}

Conclusion: The efficiency of the heterologous plant enzymes supersedes the overexpression of the native enzyme in the E. coli production system, which leads to cell death in fabF overexpression and fabB deletion mutants. The utilization of our plasmid based system would allow generation of plant like fatty acids in $E$. coli and their subsequent chemical or enzymatic conversion to high end oleochemical products.

Keywords: Type-II fatty acid synthase, Fatty acid biosynthesis, Long chain fatty acids, Heterologous expression, Chloroplast, $\beta$-Ketoacyl-[acyl carrier protein] synthase I and II, Escherichia coli, Arabidopsis thaliana

\section{Background}

To circumvent the ecological impact of plant oil production for generation of oleochemical building blocks, there is an increasing industrial demand for microbial generated fatty acids derived from bacteria, yeast or algae [1, 2] The chain length and degree of saturation of renewable fatty acids channels their use into either the biofuel, pharmaceutical or chemical industry. Wilde type E. coli produces a small range of saturated and unsaturated fatty acids with a chain length ranging from $\mathrm{C} 12$ to $\mathrm{C} 18$.

*Correspondence: brueck@tum.de

Werner Siemens-Chair of Synthetic Biotechnology, Department

of Chemistry, Technical University of Munich, 85748 Garching, Germany
Compared to other organisms it is neither a lipid accumulating organism nor a direct producer of high value fatty acid derivatives. However, due to its ease of genetic manipulation, E. coli has been successfully modified for the production for high value lipids and has been established as a model for generation of tailor made fatty acids $[1,3-7]$.

The sustainable production of microbial fatty acids and their derivatives as a renewable alternative to petroleum and other natural hydrocarbons has been the research focus over the past decades [1,3,5-10]. Escherichia coli's fatty acid biosynthesis pathway has been thoroughly investigated $[3,11,12]$. A profound understanding of the E. coli fatty acid biosynthesis pathway and the enzymes 
involved is the basis for the remodeling its biosynthetic capacity to generate tailor made fatty acid profiles $[1$, 13-17].

Prokaryotes like E. coli and prokaryote derived plant plastids both harbor the type-II fatty acid synthase (FAS), which is responsible for de-novo fatty acids biosynthesis. In $E$. coli, the FAS enzyme complex is made up of the FAB cluster, comprising the enzymes $f a b A, f a b B$, $f a b D, f a b F, f a b G, f a b H, f a b I$ and $f a b Z$. The complete FAB complex is responsible for fatty acid production from Acetyl-CoA and the subsequent elongation using Malonyl-ACP (Acyl carrier protein) (Fig. 1). The natural fatty acid distribution ranges from $\mathrm{C} 12$ to $\mathrm{C} 18$ saturated and unsaturated fatty acids, with low amounts of vaccinate (C18:1). The formation of 3-Ketoacyl-ACP is catalyzed by the 3-Ketoacyl-ACP synthases (FabH, FabB and FabF)

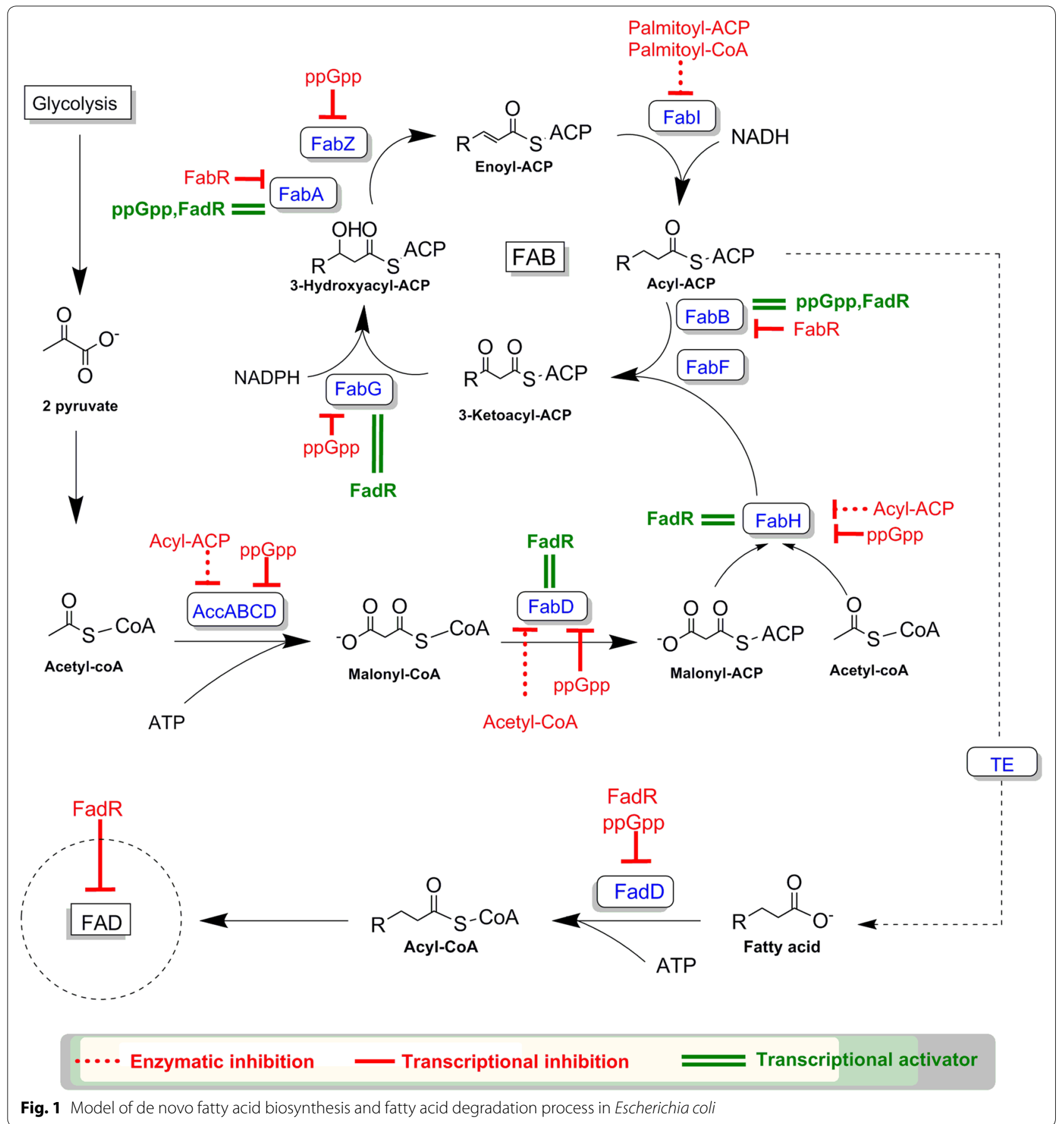


through the condensation of an acyl-ACP with malonylACP. The first condensation reaction of acetyl-CoA and Malonyl-ACP is performed by $\mathrm{FabH}$, initiating the first cycle of elongation. The proceeding two carbon elongation steps are performed solely by FabB and FabF (Fig. 1) $[1,14,18,19]$. Unlike plants, wild type $E$. coli does not possess a native desaturase [12], therefore the synthesis of unsaturated fatty acids is catalyzed anaerobically by FabA and FabB $[1,12]$. FabA is specific towards the 10-carbon acyl chain intermediate (3-hydroxydecanoyl-ACP) and introduces a double bond at the 10th carbon yielding trans-2-decenoyl-ACP which is then further isomerized by FabA to cis-3-decenoyl-ACP. FabB subsequently catalyzes the elongation of cis-3-decenoyl-ACP, which is the rate limiting step in unsaturated fatty acid synthesis. The overexpression of either FabA or FabB alone does not improve unsaturated fatty acid concentrations $[1,12]$.

Both FabB and FabF show activity towards saturated fatty acyl-ACPs up to C14, however FabB has low activity towards C14:0, while FabF only shows low activity towards C16:0 $[1,12,19]$. Regarding the synthesis of unsaturated fatty acids, FabB performs the elongation steps up to C16:1, while FabF only catalyzes the last elongation step leading to cis-vaccenic acid (C18:1). Due to the temperature sensitive nature of the FabF protein, which is expressed and active at low temperatures [20], FabB is more active and abundant $[1,12,19]$. The overexpression of FabB alone does not improve fatty acid titers and its deletion causes auxotrophy for unsaturated fatty acids. The overexpression of FabF is lethal to E. coli probably due to the high level of the enzyme both binding and inhibiting the function of FabD thus blocking the FAB cycle and increasing malonyl-CoA levels. The deletion of $f a b F$ inhibits C18:1 and C18:0 fatty acid production [1, $12,19,21,22]$.

The fatty acid biosynthesis process in $E$. coli has been extensively investigated and modified in order to increase the total fatty acid titers, shifting the fatty acid production towards a specific chain length or altering the degree of saturation of the cellular fatty acid pool. Such metabolically engineered $E$. coli strains have made use of different ACP-Thioesterases, for example, the leaderless version of the native $E$. coli TesA (the leaderless version possesses a broader substrate preference) or the Arabidopsis thaliana AtFatA [10, 23]. Most thioesterases have a wide range of activity towards ACP-fatty acid chain length with a preferred specificity towards a specific chain length. Thioesterases provide a metabolic sink by deregulating the negative feedback mechanism of ACP bound fatty acids rather than specifically altering the FAB pathway [24]. Expression levels of each thioesterase must be carefully optimized since a slight increase above the optimal concentration leads to the inhibition of FFA production [23,
25]. Other engineered strains have deleted genes of the FAD pathway in order to prevent fatty acid degradation. Specifically, the deletion of $f a d D$ (fatty-acyl-CoA ligase) or $f a d E$ the first enzyme in the fatty acid oxidation cycle inhibit fatty acid catabolism, thereby leading to intracellular fatty acid accumulation. However, the fragile balance of the FAB pathway and its strict regulation have left little or no option for manipulation.

$K A S I$ and KASII ( $\beta$-ketoacyl-[acyl carrier protein] synthase I and II) are the respective homologues of $f a b B$ and $f a b F$ in plant chloroplasts [26-28]. Both A. thaliana KAS enzymes share a mere $36 \%$ identity to their $E$. coli homologues, with the active site and catalytic triad being conserved in both species. Furthermore, KASII is not temperature sensitive as its counterpart, FabF, in E. coli [26].

Escherichia coli has an enormous in vitro potential for fatty acid synthesis, however several bottlenecks limit its in vivo production capacity $[29,30]$. In this study, we address one of the bottlenecks and report the successful manipulation of the very heavily modulated FAB elongation system in $E$. coli driving the already high flux pathway towards the production of longer chain fatty acid. We successfully implemented the ability of chloroplastic A. thaliana enzymes to complement and synergistically function with the $E$. coli FAB system in the selective production of long chain fatty acids.

\section{Results \\ Expression of KASI and KASII in wildtype BL21DE3}

Expression of both plant enzymes in wild type E. coli BL21 (DE3) resulted in an increase in the intracellular fatty acid pool. Moreover, we observed a shift in the fatty acid distribution relative to the wild type BL21 (DE3) strain harboring an empty pet28a-plasmid.

In the KASII clones, we detected an increase in unsaturated fatty acids, particularly long chain fatty acids (i.e. C16:1 and C18:1) with regard to the wildtype BL21 (DE3) strain.

By comparison, KASI clones have shown improved fatty acids concentrations with specific increases in C14:0, C16:0, C16:1, C18:0 and C18:1 fatty acids. Saturated fatty acids constituted $52 \%(\mathrm{~mol} / \mathrm{mol})$ of the total fatty acid pool in our KASI clone versus $56 \%(\mathrm{~mol} / \mathrm{mol})$ in the control (Fig. 2). Amounts of C18:1 and its cyclic propane derivative C19:0c constitute $36 \%(\mathrm{~mol} / \mathrm{mol})$ of total fatty acids in our KASI clone versus $38 \%(\mathrm{~mol} / \mathrm{mol})$ in the control. However, we have noticed a decrease in cyclopropane conversion in our KASI strain, from $13 \%$ $(\mathrm{mol} / \mathrm{mol})$ in the control down to $3 \%(\mathrm{~mol} / \mathrm{mol})$ in our clone. Moreover, palmitoleic acid constitutes up to $12 \%$ $(\mathrm{mol} / \mathrm{mol})$ of the total fatty acid pool in our KASI clone versus $6 \%(\mathrm{~mol} / \mathrm{mol})$ in the control. Notably, unsaturated 


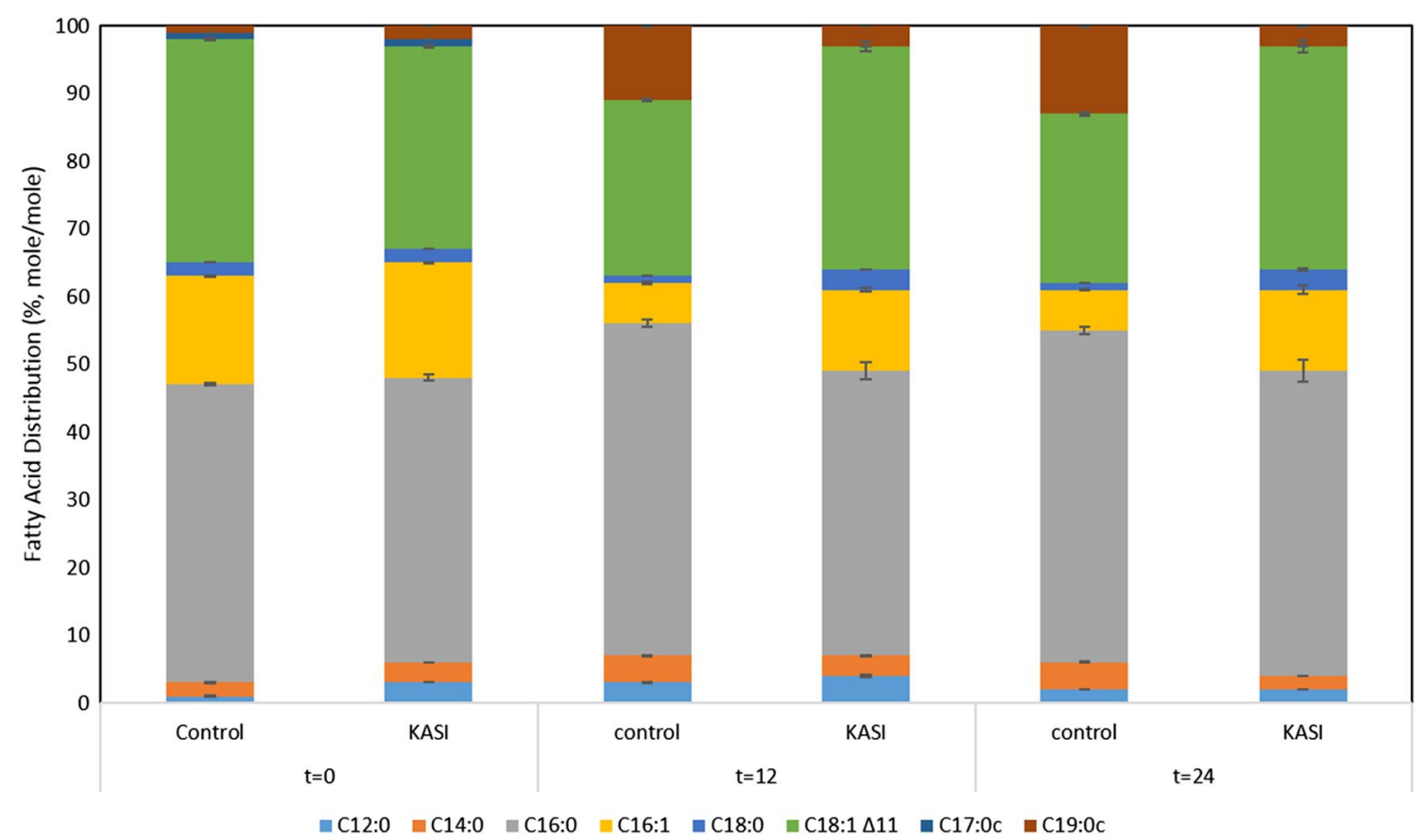

Fig. 2 Fatty acid distribution in mole percent (\% mole/mole) of "control": wild type Escherichia coli BL21 (DE3) versus "KASI": BL21 (DE3) expressing pET28aKASI at different time intervals. $T=0$ : directly before induction with IPTG, $\mathrm{t}=12: 12 \mathrm{~h}$ after induction with IPTG, $\mathrm{t}=24: 24 \mathrm{~h}$ after induction with IPTG. All fatty acid values are the average of at least three biological replicates with the associated standard deviation indicated

fatty acids constitute $44 \%(\mathrm{~mol} / \mathrm{mol})$ of total fatty acids in the wild type strain versus $48 \%(\mathrm{~mol} / \mathrm{mol})$ in the KASI clone.

\section{KASI and KASII in fadD knockouts}

In the $\triangle \mathrm{fadD}$ strain a different fatty acid distribution was observed with a slight increase in fatty acid concentrations relative to the control strain, which is in accordance with previous literature [31, 32]. Our $\Delta$ fadD KASI clone showed a significant increase in overall fatty acid accumulation (see Additional file 1: Table S3). Interestingly, cis-vaccenic acid did comprise $4 \%(\mathrm{~mol} / \mathrm{mol})$ of the total fatty acids in the $\Delta$ fadD strain compared to $21 \%$ $(\mathrm{mol} / \mathrm{mol})$ in our $\Delta$ fadD KASI clone. The total amount of generated cis-vaccenic acid including the proportion converted to C19:0c, in the $\Delta$ fadD strain amount to $17 \%$ ( $\mathrm{mol} / \mathrm{mol})$ of the total fatty acids produced versus $24 \%$ $(\mathrm{mol} / \mathrm{mol})$ in our $\Delta$ fadD KASI clone (Fig. 3). In our control strain, we observed an almost complete conversion of palmitoleic acid $(1 \% \mathrm{~mol} / \mathrm{mol})$ to its cyclopropane fatty acid form $(26 \% \mathrm{~mol} / \mathrm{mol})$. The $\Delta$ fadD KASI clone retains $18 \%(\mathrm{~mol} / \mathrm{mol})$ of the total fatty acids in the palmitoleic acid form versus $7 \%$ in the cyclopropane fatty acid form.

The $\Delta$ fadD KASII clone retains $16 \%$ of the total fatty acids in palmitoleic form versus $4 \%$ in cyclopropane form. Moreover, the fatty acid distribution in the $\Delta$ fadD KASII clone shows $30 \%$ of its total fatty acids are $\mathrm{C} 18$ versus
$23 \%$ in the $\Delta$ fadD KASI clone and $18 \%$ in the $\Delta$ fadD strain. We observe an increase in the total amounts of unsaturated fatty acids from $44 \%$ in the $\Delta$ fadD strain, to $47 \%$ in the $\Delta$ fadD KASI strain to $49 \%$ in the $\Delta$ fadD KASII strain (Fig. 3).

\section{KASII in fabF knockouts}

The generated $\Delta$ fabF strain, due to the loss of the FabF protein responsible for the elongation of 16 carbon atom acyl ACPs, showed a significant decrease in $\mathrm{C} 18$ fatty acid species (see Additional file 1: Table S4), which is in accordance with previous literature [22]. The deletion also resulted in a shift in fatty acid distribution where palmitic and palmitoleic fatty acids accumulated making up more than $70 \%$ of the total fatty acids. After the cloning of the Arabidopsis thaliana beta ketoacyl acp synthase II (KASII) gene, a significant increase of C18 fatty acid levels were observed, 3.5-fold increase compared to the $\Delta$ fabF strain, in addition to a 2.6 -fold increase in palmitoleic acid. The fatty acid distribution of our $\Delta \mathrm{fabF}$ KASII clone highly varies from that of the $\Delta$ fabF strain as seen in Fig. 4. It is noteworthy to state that C19:0c fatty acids were nonexistent in both strains, and a significant reduction in C17:0c fatty acids, from $34 \%$ in the $\Delta \mathrm{fabF}$ strain to $9 \%$ in the $\Delta$ fabF KASII clone, has been observed. No significant change in fatty acid concentration could be detected. 


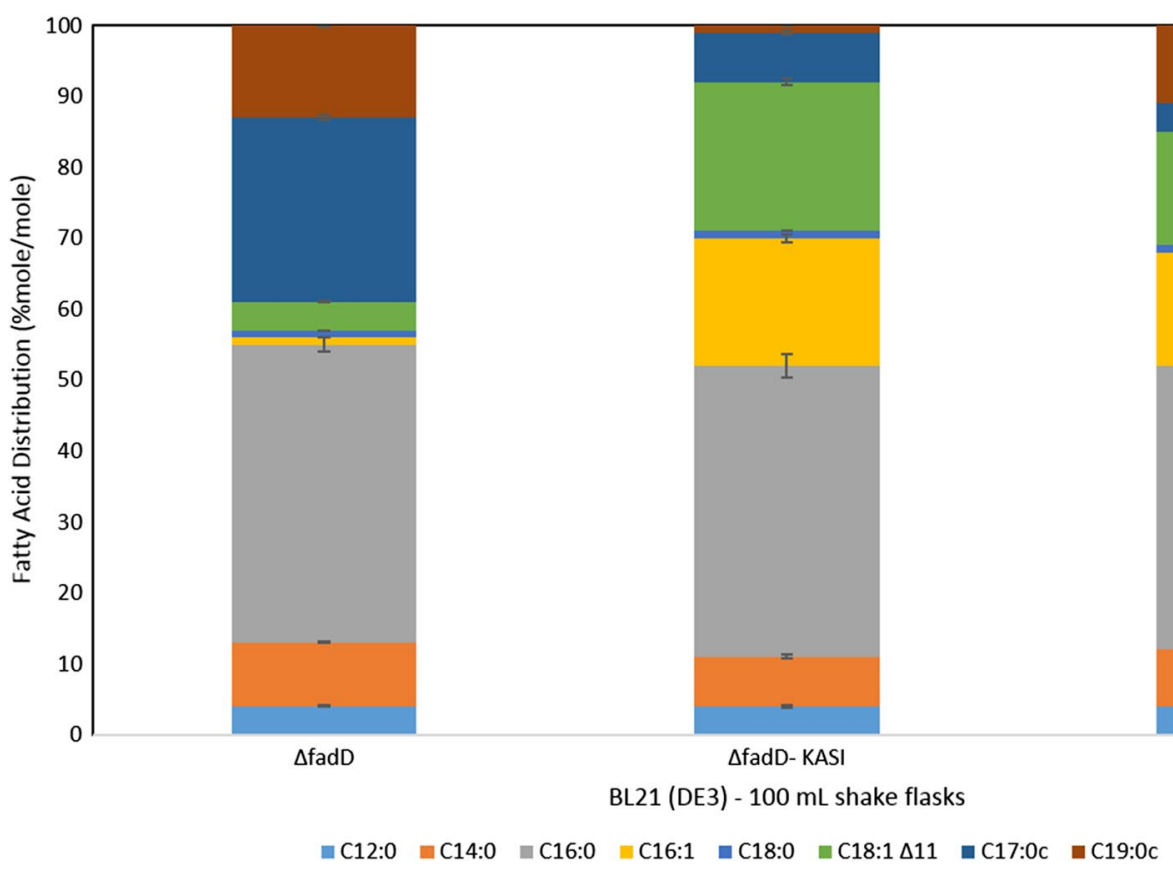

Fig. 3 Fatty acid distribution in mole percent (\% mole/mole) of E. coli BL21 (DE3) $\triangle$ fadD pET28a - versus BL21 (DE3) $\triangle$ fadD expressing pET28aKASI and pET28aKASII separately. All fatty acid values are the average of at least three biological replicates with the associated standard deviation indicated

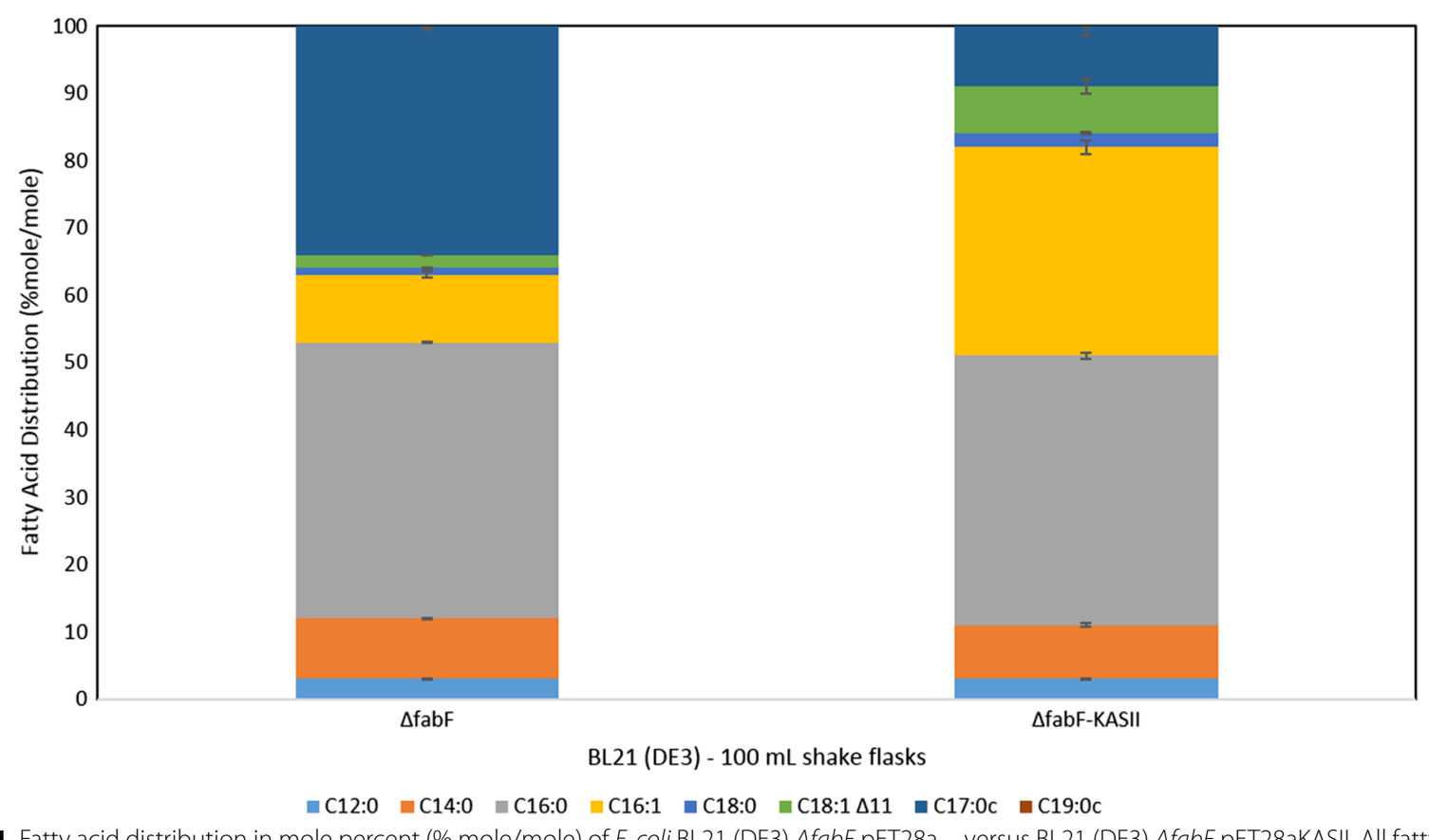

Fig. 4 Fatty acid distribution in mole percent (\% mole/mole) of E. coli BL21 (DE3) $\triangle$ fabF pET28a- versus BL21 (DE3) $\triangle$ fabF pET28aKASII. All fatty acid values are the average of at least three biological replicates with the associated standard deviation indicated 


\section{KASI in fabB knockouts}

According to literature, the deletion of $f a b B$ causes auxotrophy to saturated fatty acids and inhibits growth [20]. We attempted to knock out $f a b B$ in our BL21 (DE3) strain using the same procedure as the $f a b F$ knock out. However, we were unable to retrieve viable colonies that had the gene knocked out. We assume that $f a b B$ is crucial for survival in BL21 (DE3) even in the presence of solid media supplemented with saturated fatty acids including oleic acid.

\section{KASI and KASII fermentation}

The shake-flasks experiments described above clearly indicate that successful cloning of plant KAS enzymes and their incorporation within the FASII system is feasible in recombinant $E$. coli. To evaluate the performance of these enhanced strains at an industrial-process, fedbatch fermentations were performed in a bioreactor with defined media.

All clones were induced under nitrogen limiting conditions to enhance lipogenesis. Samples were collected after $48 \mathrm{~h}$ of induction with IPTG. Total fatty acid concentration of the KASI and KASII clone in $\triangle$ fadD strain reached $292 \mathrm{mg} / \mathrm{g}$ And $220 \mathrm{mg} / \mathrm{g}$ DCW (see Additional file 1: Table S5 and S6). In particular, $\Delta$ fadD KASI showed an $80 \%$ increase in fatty acid concentrations, while $\Delta \mathrm{fadD}$ KASII also showed a $40 \%$ increase compared to $\Delta$ fadD strain. The total amount of unsaturated fatty acids was higher in the clones expressing the KASII protein compared to all other clones (Fig. 5b).

Our fermentation results demonstrate that the $\triangle \mathrm{fabF}$ strain's C18 fatty acids constitute only $4-5 \%$ of the total fatty acids. Moreover, the KASII complemented strain shows an increase of $\mathrm{C} 18$ fatty acid species to by about
$13 \%$ with regard to the total fatty acid pool (Fig. 5a). By contrast, complementation with KASI resulted in $10 \%$ of total fatty acid species being $\mathrm{C} 18$ fatty acids. It is noteworthy that the increase of $\mathrm{C} 18$ fatty acids in the KASI strain was stearic acid, whereas the increase in the KASII strain was cis-vaccenic acid. This has been also observed in the $\Delta$ fadD strain containing KASI and KASII. Most notably, neither the increase in fatty acid concentrations nor the shift in the fatty acid profile was lethal to the cell. This is based on the fact that control and mutant strains have all exhibited almost the same growth rate with almost the same final $\mathrm{OD}_{600}$ ).

\section{Discussion}

Decades of work have been dedicated to the biochemical optimization and metabolic engineering of $E$. coli aiming to increase fatty acids yield with tailored profiles $[1,3,4$, 33]. A common approach in early metabolic engineering studies involved addressing the bottlenecks observed in the native $E$. coli FAS system and its tight regulation, optimizing precursor supply, redirecting the carbon flux into a product of interest and downregulating or eliminating competing pathways $[1,3-6,29,34]$. Despite this body of work, additional improvement in fatty acid production yield and profile specificity still requires further examination of pathway limitations and alleviation of the tight feedback regulations. However, these strategies are time-consuming and necessitate fine-tuning individual pathway components and targeting each rate-limiting factor until the desired goal is reached $[3,5,6,8,11,16]$.

In the fatty acid biosynthetic pathway of $E$. coli, both FabB and FabF proteins function in the condensation reaction and are the first enzymes in the fatty acid elongation system $[1,14]$. Their expression level and
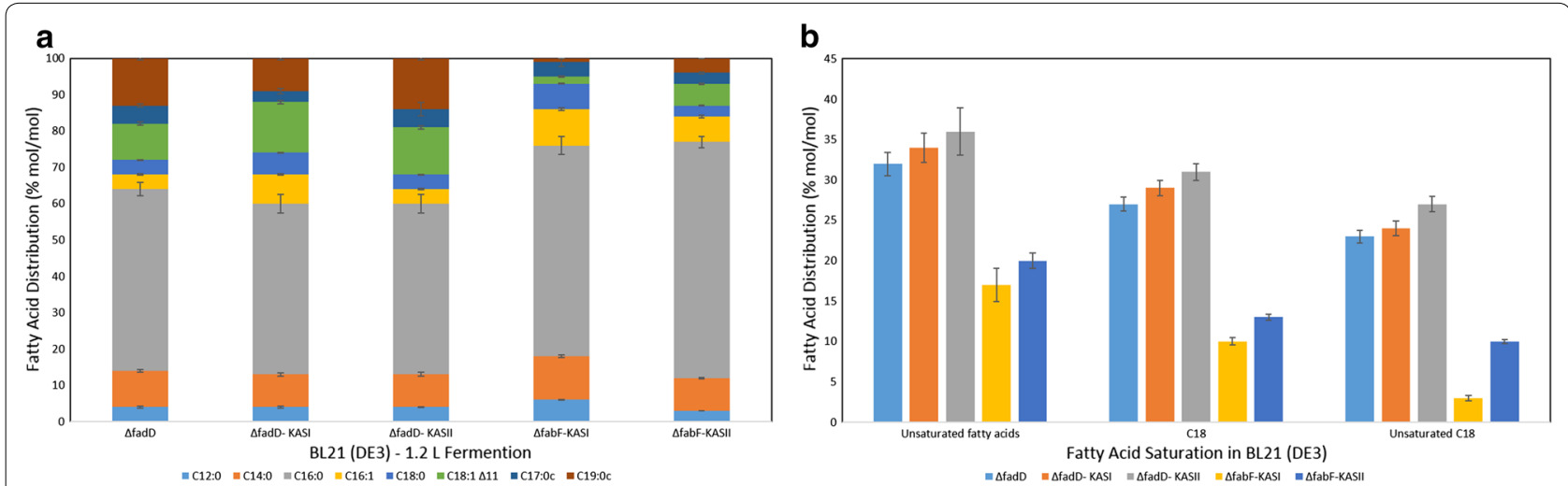

Fig. 5 a Fatty acid distribution in mole percent (\% mole/mole) of the 1.3 L fermentation of E. coli BL21 (DE3) $\triangle$ fadD expressing an empty pET28a vector, pET28aKASI and pET28aKASII respectively and of E. coli BL21 (DE3) $\triangle$ fabF expressing pET28aKASI and pET28aKASII respectively. $\mathbf{b}$ Comparison of total Unsaturated FA, total C18 FA and unsaturated C18 FA distribution (\% mole/mole) of the same clones in part $\mathbf{a}$. All fatty acid values are the average of at least three biological replicates with the associated standard deviation indicated 
specificity towards the substrate greatly affects the fatty acid profile of the organism. The FadD protein is responsible for the initial step of fatty acid degradation by adding a CoA group to a free fatty acid. In comparison, in plant chloroplasts, three isoforms of ketoacyl-ACP synthase exist, encoded by the KASI, KASII and KASIII genes $[26,27]$. The KASIII enzyme is responsible for the initial condensation reaction and accepts only acetyl-CoA as the priming unit. In contract, KASI and KASII use betaketoacyl-[acp] as the priming unit and are crucial for further elongation of the carbon chain from $\mathrm{C} 4$ to $\mathrm{C} 16$ for KASI and from C16 to C18 for KASII [26, 27].

In the present study, wild type E. coli $\mathrm{Bl} 21$ (DE3) strain was genetically modified for the purpose of enhancing the strictly modulated FASII system for the production of long chain fatty acids. KASI and KASII genes were synthesized following codon optimization for optimal expression and the endogenous $E$. coli fadD was knocked out to inhibit the degradation of free fatty acids $[1,3,4]$. We demonstrate for the first time that eukaryotic, plant KAS enzymes are functional within the native FASII system of E. coli, allowing for a definitive shift in the fatty acid profile.

Previous studies showed that the overexpression of FabB alone or in combination with other enzymes of the FAS system in $E$. coli did not improve fatty acid concentrations $[1,12]$. In this study, we demonstrate that the overexpression of the A. thaliana KASI gene, the homologue of FabB, in wild type $E$. coli shows an improvement in the overall production of saturated fatty acids with increases in myristic, palmitic acid, and stearic acid in addition to cis-vaccenic acid. We were unable to knock out the $f a b B$ gene at its locus. However, at present there is only one literature precedence that reports a $f a b B$ deletion mutant [35], which may be an indication of the difficulties to generate a knock-out at this particular locus. It is noteworthy that the lack of the $f a b B$ deletion mutant does not limit the conclusions drawn from our data.

Overexpressing the native E. coli FabF was previously reported to inhibit the FASII system and therefore fatty acid production. This lead to an intracellular accumulation of malonyl-CoA, which induced cell death [22]. FabF is reported to complex with FabD. Overexpression of FabF leads quantitative elimination of free FabD proteins in the cell, which stalls fatty acid synthesis. This hold true since FabD requires sequential complexation with FabH and FabB in order to activate the FAS system [22]. In turn, this drives malonyl-CoA accumulation, which in turn also inhibits fatty acid production and is lethal to the cell [22].

The overexpression of FabF has been widely applied to increase malonyl-CoA pools for subsequent production of polyketides and flavonoids [21]. Cloning of the $A$. thaliana KASII gene-the homologue of fabF-in E. coli was not lethal to the cell and did not inhibit the endogenous fatty acid synthase. Hence KAS II overexpression allowed fatty acid synthesis to commence without accumulation of manolyl CoA. Overexpression of KASII additionally provided an improvement in the overall fatty acid concentrations, particularly driving up the palmitoleic and cis-vaccenic acid pool. In contrast to its $E$. coli equivalent FabF [20], the $A$. thaliana KASII is active over the broad temperature range $\left(25-37^{\circ} \mathrm{C}\right)$ assayed in this study (data not shown).

In accordance with the literature, knocking out the $f a b F$ gene severely inhibited the production of $\mathrm{C} 18$ species [1]. The expression of the KASII protein in the $f a b F$ knock out strain not only complemented the loss of function of the endogenous protein, but it also leads to a significant increase in both palmitoleic and cis-vaccenic acids relative to the wild type E. coli. The expression of KASI did not complement the loss of the native $f a b F$ gene, however, it confirmed the pattern of KASI expression in other clones where significant increases in palmitoleic and stearic acid were measured.

The complementation of $\Delta \mathrm{fabF}$ strain with KASII, suggests that chloroplast enzymes, while only sharing 33\% identity with the native $E$. coli enzymes, function efficiently in the prokaryotic system. Moreover, our cumulative data suggests that the overexpression of both KASI and KASII did not induce a transcriptional inhibition by FabR.

Several studies have extensively targeted the upstream processes of the fatty acid synthase system [8]. Whether, to increase malonyl-CoA levels, the main precursor for the FAS, by targeting the $A c c A B C$ and $A c c D$ genes or by overexpressing or cloning a recombinant KASIII enzyme-the enzyme responsible for the initial precursor condensation in plants $[8,25]$. However, these systems were limited at the second condensation reaction coordinated by the FabB and FabF enzymes. The second condensation reaction is a very rapid step, where elongation is fast and reaches its limit according to what substrate each enzyme can accept. In that regard, FabB was limited at $\mathrm{C} 14$, while FabF is only limited to accept C16 fatty acid species as substrates. The use of $A$. thaliana KASI and KASII enzymes drove the elongation step beyond the acceptance of C16 fatty acids as FAS substrates. This KAS dependent feature results in a significant change in the fatty acid distribution. KAS enzyme overexpression alone is not sufficient to drastically increase fatty acid concentrations. Surprisingly; together our synergistic plant and bacterial fatty acid biosynthesis system can improve fatty acid concentrations and distributions by cumulatively enhancing both upstream and downstream fatty acid biosynthesis processes. Most notably, both beta-ketoacyl 
synthases KASI/II isolated from A. thaliana are completely compatible with the E. coli FAB pathway. It is imperative to state that both enzymes have shown to be more effective than the overexpression of the native enzyme.

\section{Conclusion}

Escherichia coli has become the model organism to study microbial fatty acid biosynthesis. However, while E. coli itself is not a dedicated oleaginous organism, extensive genetic engineering allowed for generation of respectable product titers. However, increasing the total fatty acid concentration and shifting the native fatty acid profile towards longer chain fatty acids with industrial application in the food and oleochemical industry remains a scientific challenge. This study has examined the effects of the plant derived, chloroplastic $\beta$-ketoacyl synthases (KAS) on the fatty acid biosynthesis of E. coli. We could demonstrate that KAS I and KAS II can complement the native fatty acid biosynthesis in $E$. coli and shift product profiles towards longer chain (C16-C18) type fatty acids. Interestingly, we these plant enzymes exert equivalent effects in E. coli as observed in their native plant source. In that respect, KAS I increases relative fatty acid concentrations of the cell, particularly promoting formation of C16-C18 type fatty acids. By contrast, KASII did not lead to a significant increase in the total fatty acid concentration but lead to a targeted relative increase of the C18:1, cis-vaccenic acid. Since, plant derived triglycerides mainly feature $\mathrm{C} 16-\mathrm{C} 18$ fatty acids, the integration of the plant derived KASI and II can mimic this fatty acid profile in E. coli. Therefore, utilization of our plasmid based system would allow generation of plant like fatty acids in $E$. coli and their subsequent chemical or enzymatic conversion to high end oleochemical products. One such example could be the conversion of cis-vaccenic acid to 10-hydroxy stearic acid using a dedicated vaccinate hydratase enzyme [36]. We are continuously probing and expanding our portfolio of $E$. coli variants for tailored fatty acid biosynthesis. In that regard, we focus on further extending the fatty acid chain length towards very long chain fatty acids, such as Eicosanoic acid (C20:0), Eicosenoic acid (C20:1) and Erucic acid (C22:1), which are in demand and associated with high pricing regimes in the production of cosmetics, lubricants and polymer industry $[37,38]$.

\section{Methods}

\section{Genes and plasmids}

cDNA sequences of both beta-ketoacyl-[acyl carrier protein] synthase I and II (KASI Gene ID: AT5G46290.3 and KASII Gene ID: AT1G74960.2) were obtained from The Arabidopsis Information Resource (TAIR), on www. arabidopsis.org. The chloropalstic transit peptide of both were predicted using SignalP 4.1 Server and removed from the mRNA sequence. The mature sequences were codon optimized for expression in E. coli and chemically synthesized by Eurofins Scientific. The genes were synthesized to include a $5^{\prime} \mathrm{BamHI}$ and a $3^{\prime}$ NotI restriction site that were later used to be cloned into the MCS of an empty pET28a vector. The cloned genes were confirmed by sequencing (Eurofins Scientific). All primers were synthesized by Eurofins Scientific and all plasmids were obtained from Novagen/Merk Millipore.

\section{Bacterial strains and growth conditions}

All bacterial strains used were obtained from Merk Millipore. E. coli DH5 alpha strain grown at $37{ }^{\circ} \mathrm{C}$ in Luria-Bertani medium was used for cloning and plasmid amplification. E. coli BL21 (DE3) strain was used for expression and fatty acid production. Minimal M9 media supplemented with $0.4 \%$ glucose and a $\mathrm{pH}=6.9$ was used for the shake flask studies. For shake flask studies, all clones were grown at $37{ }^{\circ} \mathrm{C}$ with the appropriate antibiotics (Kanamycin $50 \mu \mathrm{g} / \mathrm{mL}$ ) and induced at an OD600 of 0.6 with 0.05 mM IPTG (isopropyl- $\beta$-D-thiogalactopyranoside).

\section{Fatty acid methylation and analysis}

Samples taken from shake flask and fermentation studies were centrifuged and subsequently washed twice with $\mathrm{ddH}_{2} \mathrm{O}$. The samples were then lyophilized and equal amounts of dry cell weight were taken for analysis. Methanol transesterification according to the protocol of Griffiths et al. was used to directly convert dry cell biomass to fatty acid methyl esters (FAMES) $0.1 \mu \mathrm{L}$ of each sample was injected into gas chromatographflame ionization detector (GC-FID) for separation and quantification. GC-MS was performed with the Thermo Scientific $^{\mathrm{TM}}$ TRACE $^{\mathrm{TM}}$ Ultra Gas Chromatograph instrument coupled to a Thermo DSQ ${ }^{\mathrm{TM}}$ II mass spectrometer and the Triplus ${ }^{\mathrm{TM}}$ Autosampler injector. The analysis was carried out using a Stabilwax ${ }^{\circledR}$ fused silica capillary column $(30 \mathrm{~m} \times 0.25 \mathrm{~mm}$, with a film thickness of $0.25 \mu \mathrm{m})$. The analysis was performed using the following temperatures: initial column temperature $50{ }^{\circ} \mathrm{C}$, programmed to increase at a rate of $4{ }^{\circ} \mathrm{C} / \mathrm{min}$ up to a final temperature of $250{ }^{\circ} \mathrm{C}$. The carrier gas used was hydrogen at a constant flow rate of $35 \mathrm{~mL} / \mathrm{min}$. FAMEs Marine Oil Standard was used as a standard reference, containing 20 components from C14:0 until C24:1. At least three biological replicates were used for fatty acid analysis. The statistical differences and percent change presented in this manuscript are only shown for differences that were statistically significant $(\mathrm{P}<0.05)$. 


\section{Fermentation}

The DASGIP ${ }^{\circledR} 1.3 \mathrm{~L}$ parallel reactor system (Eppendorf AG) was used to perform parallel fermentations. A modified $\mathrm{M} 9$ media consisting of $8 \mathrm{~g} \mathrm{~L}^{-1} \mathrm{NH} 4 \mathrm{Cl}$, $13.3 \mathrm{~g} \mathrm{~L}^{-1} \mathrm{KH} 2 \mathrm{PO} 4,1.24 \mathrm{~g} \mathrm{~L}^{-1} \mathrm{MgSO}_{4} \cdot 7 \mathrm{H}_{2} \mathrm{O}, 0.42 \mathrm{~g} \mathrm{~L}^{-1}$ $\mathrm{FeCl}^{3-} \cdot 6 \mathrm{H}_{2} \mathrm{O}, 40 \mathrm{~g} \mathrm{~L}^{-1}$ Glucose was used as batch media. Fermenters were inoculated with an overnight pre-culture with a starting OD600 of 0.1 . The cultivation temperature was kept constant at $37{ }^{\circ} \mathrm{C}$. Initial stirring velocity and airflow was set to $200 \mathrm{rpm}$ and to 0.2 volumes of air per volume of medium per min (vvm), respectively. Dissolved oxygen was maintained at $30 \%$ by successive increases of the stirrer velocity, the oxygen concentration, and eventually the airflow. A $\mathrm{pH}$ value of 7.00 was controlled by the addition of $6 \mathrm{M}$ aqueous $\mathrm{NaOH}$. A pH value shift above 7.05 initiated a feed shot of $40 \mathrm{~mL}$. The feed solution consisted of $500 \mathrm{~g} \mathrm{~L}^{-1} \mathrm{glu}-$ cose, $20 \mathrm{~g} \mathrm{~L}^{-1} \mathrm{MgSO} 4 \cdot 7 \mathrm{H}_{2} \mathrm{O}, 2 \mathrm{mg} \mathrm{L}^{-1}$ thiamine- $\mathrm{HCl}$, $16 \mathrm{~mL} 100 \times$ trace elements solution $\left(5 \mathrm{~g} \mathrm{~L}^{-1}\right.$ EDTA; $0.83 \mathrm{~g} \mathrm{~L}^{-1} \mathrm{FeCl}^{3-} \cdot 6 \mathrm{H}_{2} \mathrm{O} ; 84 \mathrm{mg} \mathrm{L}^{-1} \mathrm{ZnCl} 2,13 \mathrm{mg} \mathrm{L}^{-1}$ $\mathrm{CuCl}^{2-} \cdot 2 \mathrm{H}_{2} \mathrm{O}, 10 \mathrm{mg} \mathrm{L}^{-1} \mathrm{CoCl}^{2-} \cdot 2 \mathrm{H}_{2} \mathrm{O}, 10 \mathrm{mg} \mathrm{L}^{-1}$ $\mathrm{H}_{3} \mathrm{BO}_{3}$, and $\left.1.6 \mathrm{mg} \mathrm{L}^{-1} \mathrm{MnCl}^{2-} \cdot 4 \mathrm{H}_{2} \mathrm{O}\right)(\mathrm{pH}=7.00)$. Samples were taken at different time points to determine the OD600. Once the clones reached limitation due to the depletion of the nitrogen source, they were induced with 0.05 mM IPTG. Samples for fatty acid analysis were collected at different intervals.

\section{Supplementary information}

Supplementary information accompanies this paper at https://doi. org/10.1186/s12934-019-1217-7.

Additional file 1: Table S1. Fatty acid distribution in $\mu \mathrm{g} / \mathrm{g}$ DCW of wild type Escherichia coli BL21 (DE3) at different time intervals. $T=0$ : directly before induction with IPTG, $\mathrm{t}=12: 12 \mathrm{~h}$ after induction with IPTG, $\mathrm{t}=24$ : $24 \mathrm{~h}$ after induction with IPTG. All fatty acid values are the average of at least three biological replicates with the associated standard deviation indicated. Table S2. Fatty acid distribution in $\mu \mathrm{g} / \mathrm{g}$ DCW of Escherichia coli BL21 (DE3) expressing pET28aKASI at different time intervals. T=0: directly before induction with IPTG, $\mathrm{t}=12$ : $12 \mathrm{~h}$ after induction with IPTG, $\mathrm{t}=24$ : $24 \mathrm{~h}$ after induction with IPTG. All fatty acid values are the average of at least three biological replicates with the associated standard deviation indicated. Table S3. Fatty acid distribution in $\mu \mathrm{g} / \mathrm{g}$ DCW of Escherichia coli BL21 (DE3) $\triangle$ fadD pET28a - versus BL21 (DE3) $\triangle$ fadD expressing pET28aKASI and pET28aKASII separately. Samples were collected $48 \mathrm{~h}$ after induction with IPTG. All fatty acid values are the average of at least three biological replicates with the associated standard deviation indicated. Table S4. Fatty acid distribution in $\mu \mathrm{g} / \mathrm{g}$ DCW of Escherichia coli BL21 (DE3) $\triangle$ fadF pET28a - versus BL21 (DE3) $\triangle$ fadD expressing pET28aKASII. Samples were collected $48 \mathrm{~h}$ after induction with IPTG. All fatty acid values are the average of at least three biological replicates with the associated standard deviation indicated. Table S5. Fatty acid distribution in $\mu \mathrm{g} / \mathrm{mg}$ DCW of the $1.3 \mathrm{~L}$ fermentation of Escherichia coli BL21 (DE3) $\triangle$ fadD expressing an empty pET28a vector, pET28aKASI and pET28aKASII respectively. Samples were collected $48 \mathrm{~h}$ after induction with IPTG. All fatty acid values are the average of at least three biological replicates with the associated standard deviation indicated. Table S6. Fatty acid distribution in $\mu \mathrm{g} / \mathrm{mg}$ DCW of the $1.3 \mathrm{~L}$ fermentation of Escherichia coli BL21 (DE3) $\triangle$ fabF expressing pET28aKASI and pET28aKASII respectively. Samples were collected $48 \mathrm{~h}$ after induction with IPTG. All fatty acid values are the average of at least three biological replicates with the associated standard deviation indicated. Table S7. Growth analysis of M9 minimal media shake flask studies of wild type Escherichia coli BL21 (DE3) expressing pET28a-, pET28aKASI and pET28aKASII respectively. Absorbance values (OD600) are the average of at least three biological replicates. Figure S1. Growth curve of M9 minimal media shake flask studies of wild type Escherichia coli BL21 (DE3) expressing pET28a-, pET28aKASI and pET28aKASII respectively. Absorbance values (OD600) are the average of at least three biological replicates with the associated standard deviation indicated.

\section{Abbreviations}

FA: fatty acid; FAB: fatty acid biosynthesis; FAS: fatty acid synthase; DCW: dry cell weight; ACP: acyl carrier protein; CoA: coenzyme A; KASI: $\beta$-ketoacyl-[acyl carrier protein] synthase I; KASII: $\beta$-ketoacyl-[acyl carrier protein] synthase II; FabB: 3-oxoacyl-[acyl-carrier-protein] synthase 1; FabF: 3-oxoacyl-[acyl-carrierprotein] synthase 2; FadD: fatty acyl-CoA ligase.

\section{Acknowledgements}

TB gratefully acknowledges funding by the Werner Siemens Foundation for establishing the field of Synthetic Biotechnology at the Technical University of Munich (TUM).

\section{Authors' contributions}

Conceived the project: EK and TB. Designed and performed the experiments: EK. Analyzed the data: EK and MH. Prepared the manuscript: EK, MF, NM and TB. Supervised the whole work: TB, MF, and NM. All authors read and approved the final manuscript.

\section{Funding}

TB and MF would like to acknowledge the financial support of the German Ministry for Education and Research (BMBF) with the Grant Numbers 031 A305A and 033RC012B. TB and NM would like to acknowledge the financial support of the Bavarian Ministry of Economic Affairs, Energy and Technology with the Grant Number (1340/68351/13/2013).

\section{Availability of data and materials}

All data generated or analyzed during this study are included in this published article and its Additional file. Additional data required is available from the corresponding author on reasonable request.

\section{Ethics approval and consent to participate}

Not applicable.

\section{Competing interests}

The authors declare that they have no competing interests.

Received: 23 May 2019 Accepted: 24 September 2019

Published online: 03 October 2019

\section{References}

1. Janssen HJ, Steinbuchel A. Fatty acid synthesis in Escherichia coli and its applications towards the production of fatty acid based biofuels. Biotechnol Biofuels. 2014;7(1):7.

2. Lu C, Napier JA, Clemente TE, Cahoon EB. New frontiers in oilseed biotechnology: meeting the global demand for vegetable oils for food, feed, biofuel, and industrial applications. Curr Opin Biotechnol. 2011:22(2):252-9.

3. Cao Y, Cheng T, Zhao G, Niu W, Guo J, Xian M, et al. Metabolic engineering of Escherichia coli for the production of hydroxy fatty acids from glucose. BMC Biotechnol. 2016;16:26.

4. Guo D, Pan H, Li X. Metabolic engineering of Escherichia coli for production of biodiesel from fatty alcohols and acetyl-CoA. Appl Microbiol Biotechnol. 2015;99(18):7805-12.

5. Handke P, Lynch SA, Gill RT. Application and engineering of fatty acid biosynthesis in Escherichia coli for advanced fuels and chemicals. Metab Eng. 2011;13(1):28-37. 
6. Kalscheuer R, Stoveken T, Luftmann H, Malkus U, Reichelt R, Steinbuchel A. Neutral lipid biosynthesis in engineered Escherichia coli: jojoba oil-like wax esters and fatty acid butyl esters. Appl Environ Microbiol. 2006;72(2):1373-9.

7. Scaglia B, Cassani E, Pilu R, Adani F. Expression of Arabidopsis thaliana S-ACP-DES3 in Escherichia coli for high-performance biodiesel production. RSC Adv. 2014;4(108):63387-92.

8. Pfleger BF, Gossing M, Nielsen J. Metabolic engineering strategies for microbial synthesis of oleochemicals. Metab Eng. 2015;29:1-11.

9. Schirmer A, Rude MA, Li X, Popova E, del Cardayre SB. Microbial biosynthesis of alkanes. Science. 2010;329(5991):559-62.

10. Zheng YN, Li LL, Liu Q, Yang JM, Wang XW, Liu W, et al. Optimization of fatty alcohol biosynthesis pathway for selectively enhanced production of C12/14 and C16/18 fatty alcohols in engineered Escherichia coli. Microb Cell Fact. 2012;11:65.

11. Cao Y, Liu W, Xu X, Zhang H, Wang J, Xian M. Production of free monounsaturated fatty acids by metabolically engineered Escherichia coli. Biotechnol Biofuels. 2014;7:59.

12. Cao Y, Yang J, Xian M, Xu X, Liu W. Increasing unsaturated fatty acid contents in Escherichia coli by coexpression of three different genes. Appl Microbiol Biotechnol. 2010;87(1):271-80.

13. Beld J, Abbriano R, Finzel K, Hildebrand M, Burkart MD. Probing fatty acid metabolism in bacteria, cyanobacteria, green microalgae and diatoms with natural and unnatural fatty acids. Mol BioSyst. 2016;12(4):1299-312.

14. Beld J, Lee DJ, Burkart MD. Fatty acid biosynthesis revisited: structure elucidation and metabolic engineering. Mol BioSyst. 2015;11(1):38-59.

15. Wang $X$, Xing $X, M a$ Q. Boosting the hydroxy fatty acid synthesis in Escherichia coli by expression of Bacillus megaterium glucose dehydrogenase. Biotechnol Biotechnol Equip. 2016;30(5):879-84.

16. Xu P, Gu Q, Wang W, Wong L, Bower AG, Collins CH, et al. Modular optimization of multi-gene pathways for fatty acids production in E. coli. Nat Commun. 2013:4:1409.

17. Zha W, Rubin-Pitel SB, Shao Z, Zhao H. Improving cellular malonylCoA level in Escherichia coli via metabolic engineering. Metab Eng. 2009;11(3):192-8.

18. Lee S, Jeon E, Yun HS, Lee J. Improvement of fatty acid biosynthesis by engineered recombinant Escherichia coli. Biotechnol Bioprocess Eng. 2011;16(4):706-13.

19. Edwards P, Nelsen JS, Metz JG, Dehesh K. Cloning of the fabF gene in an expression vector and in vitro characterization of recombinant fabF and fabB encoded enzymes from Escherichia coli. FEBS Lett. 1997;402(1):62-6.

20. Garwin JL, Klages AL, Cronan JE Jr. Beta-ketoacyl-acyl carrier protein synthase II of Escherichia coli. Evidence for function in the thermal regulation of fatty acid synthesis. J Biol Chem. 1980;255(8):3263-5.

21. Cao W, Ma W, Zhang B, Wang X, Chen K, Li Y, et al. Improved pinocembrin production in Escherichia coli by engineering fatty acid synthesis. J Ind Microbiol Biotechnol. 2016;43(4):557-66.

22. Subrahmanyam S, Cronan JE Jr. Overproduction of a functional fatty acid biosynthetic enzyme blocks fatty acid synthesis in Escherichia coli. J Bacteriol. 1998;180(17):4596-602.

23. Lu X, Vora H, Khosla C. Overproduction of free fatty acids in E. coli: implications for biodiesel production. Metab Eng. 2008;10(6):333-9.
24. Jing F, Cantu DC, Tvaruzkova J, Chipman JP, Nikolau BJ, Yandeau-Nelson $M D$, et al. Phylogenetic and experimental characterization of an acyl-ACP thioesterase family reveals significant diversity in enzymatic specificity and activity. BMC Biochem. 2011;12:44.

25. Davis MS, Solbiati J, Cronan JE Jr. Overproduction of acetyl-CoA carboxylase activity increases the rate of fatty acid biosynthesis in Escherichia coli. J Biol Chem. 2000;275(37):28593-8.

26. Hakozaki H, Park JI, Endo M, Takada Y, Kazama T, Takeda Y, Suzuki G, Kawagishi-Kobayashi M, Watanabe M. Expression and developmental function of the 3-ketoacyl-ACP synthase 2 gene in Arabidopsis thaliana. Genes Genet Syst. 2008;83:10.

27. Wu GZ, Xue HW. Arabidopsis beta-ketoacyl-[acyl carrier protein] synthase $i$ is crucial for fatty acid synthesis and plays a role in chloroplast division and embryo development. Plant Cell. 2010;22(11):3726-44.

28. Guan X, Okazaki Y, Lithio A, Li L, Zhao X, Jin H, et al. Discovery and characterization of the 3-hydroxyacyl-ACP dehydratase component of the plant mitochondrial fatty acid synthase system. Plant Physiol. 2017;173(4):2010-28.

29. Liu T, Vora H, Khosla C. Quantitative analysis and engineering of fatty acid biosynthesis in E. coli. Metab Eng. 2010:12(4):378-86.

30. Liu Z, Zhang Y, Jia X, Hu M, Deng Z, Xu Y, et al. In vitro reconstitution and optimization of the entire pathway to convert glucose into fatty acid. ACS Synth Biol. 2017;6(4):701-9.

31. Li M, Zhang X, Agrawal A, San KY. Effect of acetate formation pathway and long chain fatty acid CoA-ligase on the free fatty acid production in E. coli expressing acy-ACP thioesterase from Ricinus communis. Metab Eng. 2012;14(4):380-7.

32. Wu H, San KY. Efficient odd straight medium chain free fatty acid production by metabolically engineered Escherichia coli. Biotechnol Bioeng. 2014;111(11):2209-19.

33. Tee TW, Chowdhury A, Maranas CD, Shanks JV. Systems metabolic engineering design: fatty acid production as an emerging case study. Biotechnol Bioeng. 2014;111(5):849-57.

34. Tan Z, Yoon JM, Chowdhury A, Burdick K, Jarboe LR, Maranas CD, et al. Engineering of $E$. coli inherent fatty acid biosynthesis capacity to increase octanoic acid production. Biotechnol Biofuels. 2018;11:87.

35. Nosho K, Yasuhara K, Ikehata Y, Mii T, Ishige T, Yajima S, et al. Isolation of colonization-defective Escherichia coli mutants reveals critical requirement for fatty acids in bacterial colony formation. Microbiology. 2018;164(9):1122-32.

36. Engleder $\mathrm{M}$, Pichler $\mathrm{H}$. On the current role of hydratases in biocatalysis. Appl Microbiol Biotechnol. 2018;102(14):5841-58.

37. Nieschlag HJ, Wolff IA. Industrial uses of high erucic oils. J Am Oil Chem Soc. 1971;48(11):723-7.

38. Leonard EC. High-erucic vegetable oils. Ind Crops Prod. 1992;1:119-23.

\section{Publisher's Note}

Springer Nature remains neutral with regard to jurisdictional claims in published maps and institutional affiliations.
Ready to submit your research? Choose BMC and benefit from:

- fast, convenient online submission

- thorough peer review by experienced researchers in your field

- rapid publication on acceptance

- support for research data, including large and complex data types

- gold Open Access which fosters wider collaboration and increased citations

- maximum visibility for your research: over 100M website views per year

At BMC, research is always in progress.

Learn more biomedcentral.com/submissions 